\title{
Low alkaline contamination bottom antireflective coatings for both 193- and 157-nm lithography applications
}

\author{
H.L. Chen ${ }^{\mathrm{a}, *}$, Y.F. Chuang ${ }^{\mathrm{b}}$, C.C. Lee ${ }^{\mathrm{b}}$, C.I. Hsieh ${ }^{\mathrm{a}}$, F.H. Ko ${ }^{\mathrm{a}}$, L.A. Wang ${ }^{\mathrm{c}}$ \\ ${ }^{a}$ National Nano Device Laboratory, 1001-1 Ta Hsueh Road Hsinchu, Hsinchu 300, Taiwan, ROC \\ ${ }^{\mathrm{b}}$ Institute of Optical Science, National Central University, Chung-Li, Taiwan, ROC \\ ${ }^{\mathrm{c}}$ Institute of Electro-Optical Engineering, National Taiwan University, Taipei, Taiwan, ROC
}

\begin{abstract}
A bilayer bottom antireflective coating (BARC) structure composed of TEOS oxide and silicon nitride film stacks is demonstrated for both $\operatorname{ArF}(193 \mathrm{~nm})$ and $\mathrm{F}_{2}(157 \mathrm{~nm})$ excimer laser lithography. The top TEOS oxide film is an $\mathrm{NH}_{3}$-contaminant-free material that can be used as an $\mathrm{NH}_{3}$ capping layer. After an oxygen plasma treatment, the bilayer structure is shown to have high thermal stability by thermal desorption spectrometry (TDS). The measured swing effect is significantly reduced by adding the bilayer BARC structure. This BARC structure could also reduce the reflectance of various highly reflective substrates to less than $2 \%$ for both 193 and $157 \mathrm{~nm}$.
\end{abstract}

(C) 2003 Elsevier Science B.V. All rights reserved.

Keywords: Bottom antireflective coatings; Low alkaline contamination; $193 \mathrm{~nm}$ lithography; $157 \mathrm{~nm}$ lithography; Bilayer structure

\section{Introduction}

According to the recent International Technology Roadmap for Semiconductors, ArF and $\mathrm{F}_{2}$ excimer laser lithographies combined with resolution enhancement techniques would lead integrated circuit (IC) technologies to generations of 90 and $65 \mathrm{~nm}$, respectively [1]. The problems of critical dimension control caused by highly reflective substrates are more serious for sub-100 nm optical lithography. It is therefore important to find a high-performance antireflective coating layer working in deep and vacuum ultraviolet spectral regimes [2].

Chemically amplified resists (CAR) with high sensitivity have been widely used in deep ultraviolet lithography and still will be used in $\mathrm{ArF}$ and $\mathrm{F}_{2}$ lithography for high-throughput applications [3]. One

\footnotetext{
*Corresponding author. Tel.: + 86-3-5726-100; fax: + 886-3-5722-715.

E-mail address: hlchen@ndl.gov.tw (H.L. Chen).
} 
of the most serious problems in chemically amplified resists is that their profile is degraded by airborne and substrate alkaline contaminants such as amine and ammonia [4]. The airborne contamination results in T-top effects and the alkaline substrate contamination results in footing effects. The T-top effect can be removed by a chemical filter. Adding capping layers or pretreating the substrate would reduce the footing effect [5]. Nitride-based (silicon nitride, silicon oxynitride) films have been demonstrated to have suitable optical characteristics for both $\mathrm{KrF}$ and ArF lithography BARC applications [6,7]. However, nitride films that result in ammonia outgassing would cause footing effects during the post-exposure bake (PEB) procedure.

In this study, we demonstrate a bilayer BARC for both ArF and $\mathrm{F}_{2}$ lithography. The BARC is composed of a TEOS oxide/silicon nitride film stack deposited by the conventional PECVD process. Silicon nitride films with optical properties suitable for BARC applications but with high $\mathrm{NH}_{3}$ contamination would degrade resist profiles. A TEOS oxide film that is $\mathrm{NH}_{3}$-contaminant-free can be used as an $\mathrm{NH}_{3}$ capping layer, and has been widely used in IC processes by conventional deposition methods. The extinction coefficient $(k)$ of a TEOS oxide film is small in 193- and 157-nm regimes, and is suitable for use as the top layer of bilayer BARC structures [8]. The swing effects of resists coated on silicon film substrates before and after adding a bilayer BARC structure are compared. We also discuss the practicability of using the TEOS-oxide and silicon nitride-based bilayer BARC for various highly reflective substrates.

\section{Experimental setup}

The reflectance spectra were measured by using a deep and vacuum ultraviolet optical spectrometer. The TEOS oxide and silicon nitride films were deposited by employing a conventional PECVD system (Surface Technology System, Multiplex Cluster). The oxygen plasma treatment of BARC films was also carried out in this PECVD system. A thermal stability test was performed with a thermal desorption spectroscope (Hitachi, UG21) to observe if there was base contamination from BARC films during the PEB procedure. The optical constants of the films were obtained by the R-T method using the N\&K analyzer $[9,10]$. The optimal thicknesses of BARC films are obtained by using optical thin-film simulation [11]. Resists used for measuring swing effects at 193 and $157 \mathrm{~nm}$ are DHA-1000 (Dongjin Semichem) and hydrogen silsesquioxane (HSQ), respectively.

\section{Results and discussion}

\subsection{Bilayer BARC structure}

By using a graded-absorption multilayer structure, BARC can be applied to various reflective substrates. In a multilayer BARC, the extinction coefficient of each BARC layer is gradually increased layer by layer, and is highest at the bottom. For convenience in the deposition process, the number of layers should be as few as possible. Here we demonstrate a bilayer BARC structure for both 193- and 157-nm lithographies. As schematically shown in Fig. 1, the BARC is composed of a TEOS oxide/silicon nitride film stack deposited by the conventional PECVD process. The high- 


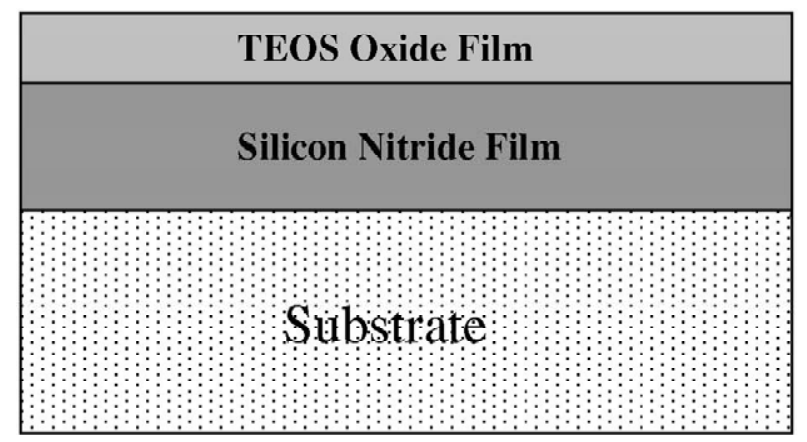

Fig. 1. Schematic of a bilayer BARC structure.

absorption silicon nitride film is as the bottom layer, and the low absorption TEOS oxide film is as the top layer of the bilayer structure.

\subsection{Thermal stability of TEOS oxide and silicon nitride films}

The contamination of TEOS oxide and silicon nitride-based BARC film was evaluated by thermal desorption spectroscopy. Fig. 2 shows the desorption curves for silicon nitride films covered by TEOS oxide films of different thicknesses. We found that the desorption curves are similar to the substrate background at TEOS-oxide film thickness of $75 \mathrm{~nm}$. For etching process consideration, the thickness of the BARC layer should be as small as possible. Therefore, the thickness requirement of the TEOS oxide films was reduced by using the oxygen plasma treatment. The oxygen gas flow rate was 100 sccm, the RF power was $50 \mathrm{~W}$, and the time period was 5 min. As shown in Fig. 2, after the oxygen

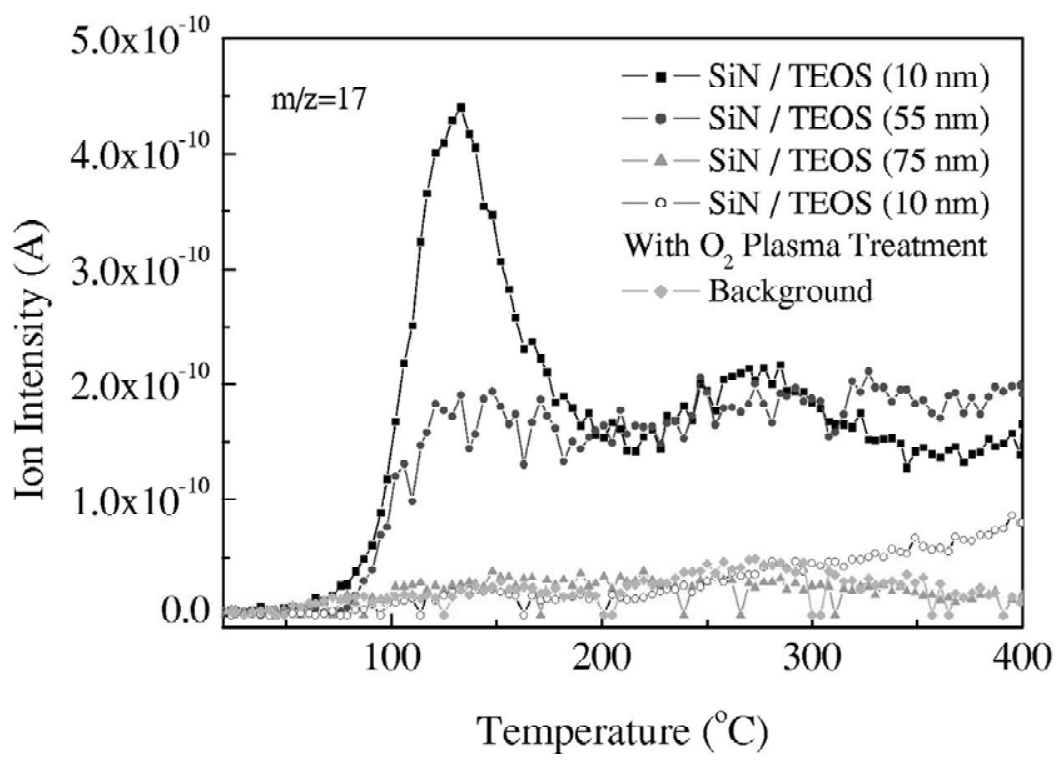

Fig. 2. $\mathrm{NH}_{3}$ desorption curves showing dependence of capped TEOS oxide films on thickness. 
plasma treatment, the $\mathrm{NH}_{3}$ desorption curves are similar to substrate background when the TEOS oxide film thickness of is $10 \mathrm{~nm}$. This is due to the capped TEOS oxide film becoming dense after the oxygen plasma treatment. Therefore, the thickness requirement of the TEOS oxide films can be reduced to $10 \mathrm{~nm}$, which makes the film suitable as the top layer of a bilayer BARC structure.

Fig. 3 shows $\mathrm{NH}_{3}$ desorption curves indicating the dependence of the oxygen plasma treatment procedure on time. When the plasma treatment time increased, the $\mathrm{NH}_{3}$ desorption intensity first decreased rapidly and then saturated. This is due to the capped TEOS-oxide film becoming dense after the oxygen plasma treatment. Results indicate that the $\mathrm{NH}_{3}$ desorption curves are similar to substrate background when the time of plasma treatment exceeded 3 min.

\subsection{Bilayer BARC structure for both 193 and $157 \mathrm{~nm}$}

After optical thin-film simulation [11], the TEOS oxide and silicon nitride films with suitable optical characteristics at 193 and $157 \mathrm{~nm}$ were chosen as constituents of the bilayer structure. The optical constants (refractive index, extinction coefficient) of TEOS oxide and silicon nitride film at $157 \mathrm{~nm}$ are $(1.660,0.054)$, and $(1.774,0.627)$, respectively. And the optical constants of TEOS oxide and silicon nitride film at $193 \mathrm{~nm}$ are $(1.562,0.035)$, and $(2.534,0.303)$, respectively. The optimal thicknesses of TEOS oxide and silicon nitride films 15 and $45 \mathrm{~nm}$ were chosen to reduce the reflectance at air/substrate interface. The reflectance of a silicon substrate was measured as about $60 \%$ at $157 \mathrm{~nm}$, as shown in Fig. 4. By adding an optimized bilayer BARC structure, the reflectance of less than $2 \%$ at both 193 and $157 \mathrm{~nm}$ can be achieved.

The bilayer BARC was added to reduce the reflectance between a silicon crystal substrate and a resist interface. The optimal thicknesses of TEOS oxide and silicon nitride films 25 and $49 \mathrm{~nm}$ were chosen to reduce the reflectance at resist/substrate interface. The simulated reflectance at 193 and 157 $\mathrm{nm}$ and can be reduced to 0.107 and $1.440 \%$, respectively. The reflectance swing curves at $193 \mathrm{~nm}$

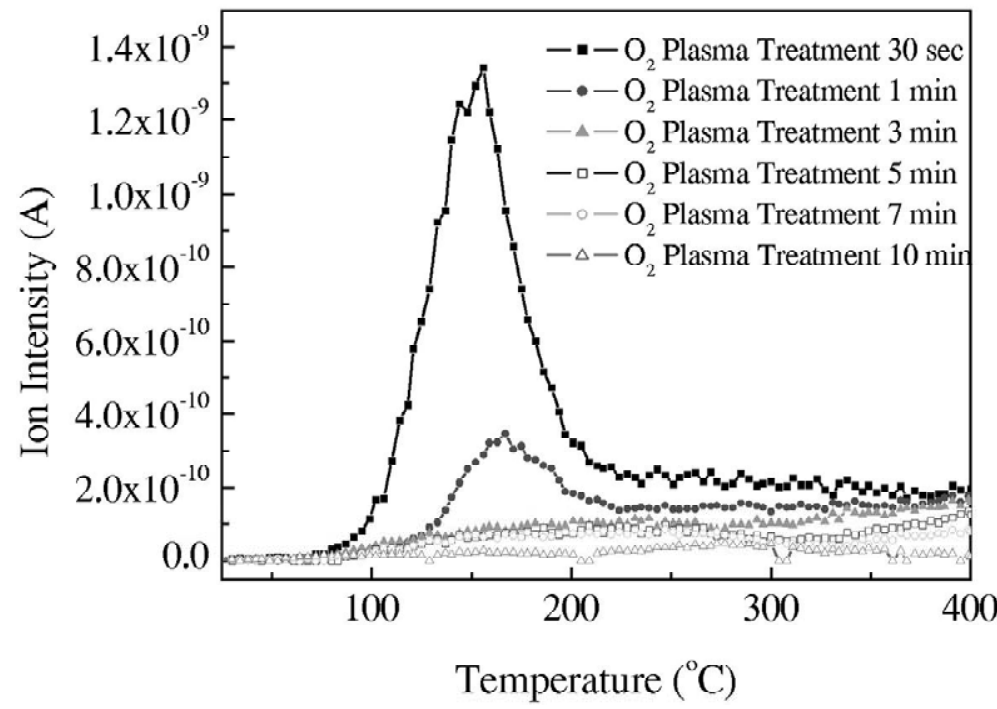

Fig. 3. $\mathrm{NH}_{3}$ desorption curves showing dependence of the oxygen plasma treatment procedure on time. 


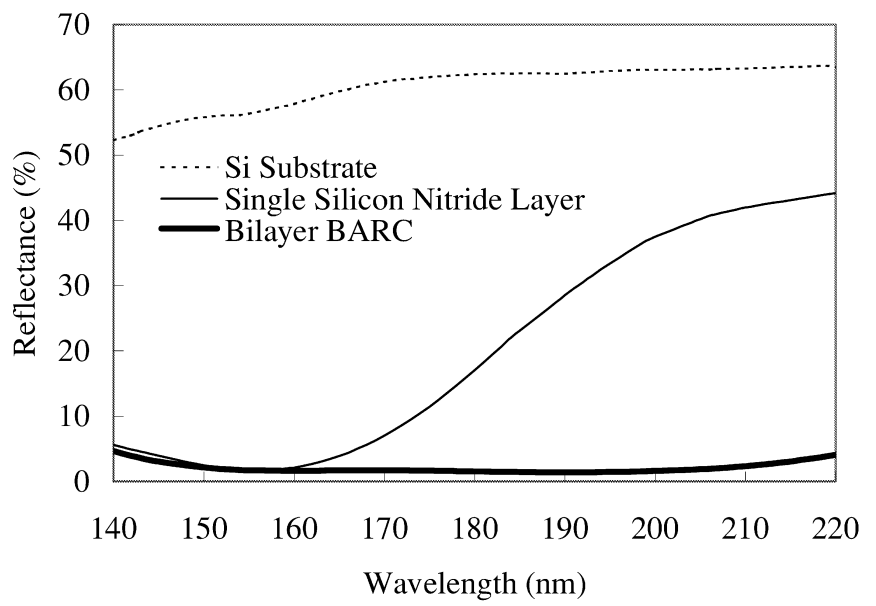

Fig. 4. Reflection spectra of a silicon substrate before and after adding single and bilayer BARC structures.

with and without a BARC layer were simulated and measured, and the results are shown in Fig. 5. The reflectance exhibits a sinusoidal variation from about 5 to $40 \%$ for the resist thicknesses range from 300 to $600 \mathrm{~nm}$ before adding the BARC layer, and the variation is reduced to about 4 to $10 \%$ after the addition.

Similarly, The reflectance swing curves at $157 \mathrm{~nm}$ with and without a BARC layer were simulated and measured, and the results are shown in Fig. 6. The reflectance exhibits a sinusoidal variation from about 30 to $65 \%$ for the resist thicknesses range from 200 to $350 \mathrm{~nm}$ before adding the BARC layer, and the variation is reduced to about $4-10 \%$ after the addition. The results indicate that the bilayer BARC can significantly reduce the swing effect incurred by a resist for both 193- and 157-nm lithography. It is thus expected that by using such a bilayer BARC, the capability of control of critical dimensions can be improved.

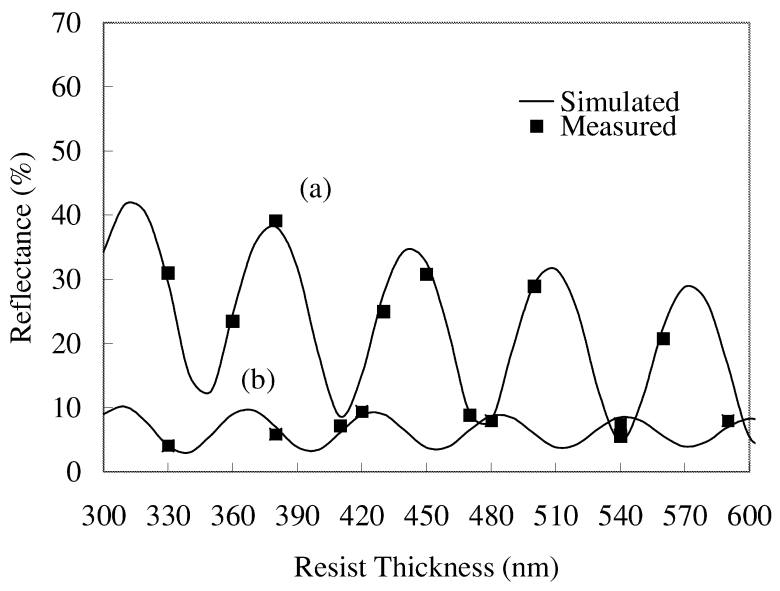

Fig. 5. Reflectance swing curves of the DHA-1000 resist coated on a silicon wafer (a) without (b) with a bilayer BARC structure at $193 \mathrm{~nm}$. 


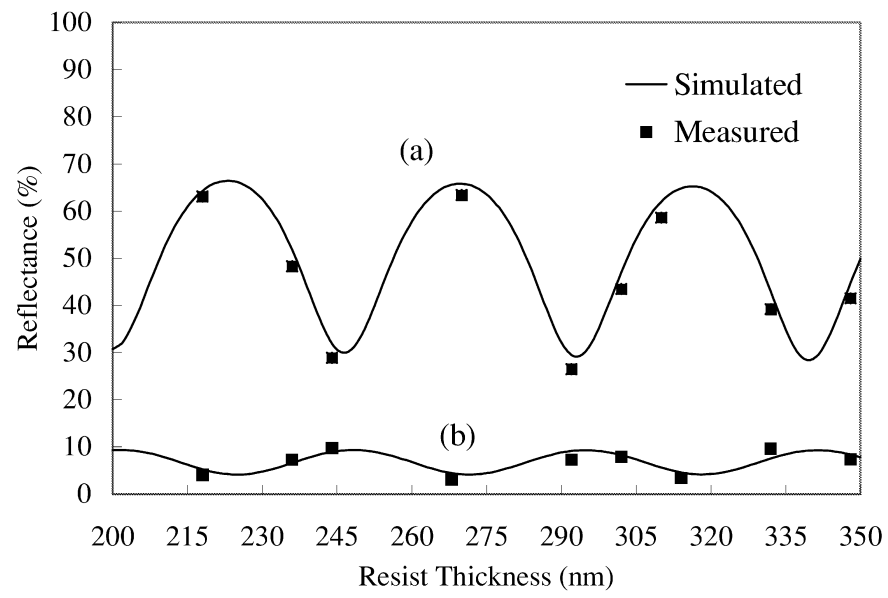

Fig. 6. Reflectance swing curves for the HSQ coated on a silicon wafer (a) before, and (b) after deposition of a bilayer BARC structure.

\subsection{Bilayer BARC structure for various highly reflective substrates}

The same bilayer $(25 / 49 \mathrm{~nm})$ structure was used to demonstrate the practicality of using TEOS oxide- and silicon nitride-based bilayer BARC structure for various highly reflective substrates such as silicon, tungsten, aluminum, copper, and platinum, which are commonly used in IC processes. These substrates are evaluated by simulating their optical performance and the results are summarized in Table 1. Results indicate this bilayer BARC structure would reduce the reflectance from the interface of the resist/high reflective materials to less than $3 \%$ at both 193 and $157 \mathrm{~nm}$. The TEOS oxide- and silicon nitride-based bilayer BARC structure is therefore expected to have great potential for use with highly reflective materials.

Table 1

A bilayer BARC structure for highly reflective substrates at 193 and $157 \mathrm{~nm}$

\begin{tabular}{|c|c|c|c|c|c|}
\hline Substrates & $\mathrm{Si}$ & $\mathrm{W}$ & $\mathrm{Al}$ & $\mathrm{Cu}$ & $\mathrm{Pt}$ \\
\hline $\begin{array}{l}\text { Optical constants at } \\
193 \mathrm{~nm}\end{array}$ & $(0.78,2.46)$ & $(1.07,2.18)$ & $(0.11,2.17)$ & $(0.95,1.37)$ & $(1.32,1.28)$ \\
\hline $\begin{array}{l}\text { Reflectance from resist/ } \\
\text { substrate at } 193 \mathrm{~nm}(\%)\end{array}$ & 56.84 & 41.80 & 90.69 & 27.14 & 16.70 \\
\hline $\begin{array}{l}\text { Reflectance after adding a } \\
\text { bilayer BARC structure at } \\
193 \mathrm{~nm}(\%)\end{array}$ & 0.107 & 0.540 & 0.573 & 0.939 & 1.071 \\
\hline Optical constants at $157 \mathrm{~nm}$ & $(0.66,2.03)$ & $(0.93,2.00)$ & $(0.08,1.73)$ & $(1.03,1.03)$ & $(1.46,1.16)$ \\
\hline $\begin{array}{l}\text { Reflectance from resist/ } \\
\text { substrate at } 157 \mathrm{~nm} \mathrm{( \% )}\end{array}$ & 53.76 & 42.10 & 91.19 & 17.67 & 12.86 \\
\hline $\begin{array}{l}\text { Reflectance after adding a } \\
\text { bilayer BARC structure at } \\
157 \mathrm{~nm}(\%)\end{array}$ & 1.440 & 1.391 & 1.424 & 2.322 & 2.783 \\
\hline
\end{tabular}




\section{Conclusions}

A bilayer BARC structure composed of TEOS oxide and silicon nitride film is demonstrated for both $\mathrm{ArF}$ and $\mathrm{F}_{2}$ excimer laser lithography. The top TEOS oxide film is an $\mathrm{NH}_{3}$-contaminant-free material that can be used as an $\mathrm{NH}_{3}$ capping layer, which is also part of the bilayer structure. After an oxygen plasma treatment, the thickness requirement of capped TEOS oxide films can be reduced from 75 to $10 \mathrm{~nm}$. The optimal thicknesses of TEOS oxide and silicon nitride films in the bilayer structure are 25 and $49 \mathrm{~nm}$, respectively. Therefore, the bilayer structure after an oxygen plasma treatment is shown to have high thermal stability by thermal desorption spectrometry. The measured swing effect is to be significantly reduced by adding a bilayer BARC on a silicon crystal substrate. This structure also has great potential used as a BARC layer on various high-reflectance substrates commonly used in IC processes.

\section{Acknowledgements}

The authors are very grateful to the National Science Council, Taiwan, ROC for supporting the NSC91-2722-2-317-200 project.

\section{References}

[1] Semiconductor Industry Association, International Technology Roadmap for Semiconductor 2001 Updated (SIA, 2001).

[2] J. Sturtevant, B. Roman, Microlithogr. World 4 (1995) 13-21.

[3] H. Ito, G.M. Wallraff, N. Fender, P.J. Brock, W.D. Hinsberg, A. Mahorowala, C.E. Larson, H.D. Truong, G. Breyta, R.D. Allen, J. Vac. Sci. Technol. B 19 (6) (2001) 2678.

[4] Y. Kawai, A. Tanaka, T. Matsuda, Jpn. J. Appl. Phys. (B) 33 (12) (1994) 7023.

[5] C.P. Soo, S. Valiyaveettil, A. Huan, A. Wee, T.C. Ang, M.H. Fan, A.J. Bourdillon, L.H. Chan, IEEE Trans. Semiconduct. Manufact. 12 (4) (1999) 462.

[6] T.P. Ong, B. Roman, Symposium on VLSI Technology Digest of Technical Papers, 73 (1995).

[7] Y. Trouiller, N. Buffet, Proc. SPIE 3333 (1998) 324.

[8] H.L. Chen, W.C. Chao, F.H. Ko, T.C. Chu, T.Y. Huang, J. Microlithogr. Microfabrication Microsyst. 1 (1) (2002) 58.

[9] A.R. Forouhi, I. Bloomer, Phys. Rev. B 34 (1988) 1865.

[10] T.C. Paulick, Appl. Opt. 25 (1986) 562.

[11] H.A. Macleod, in: Thin Film Optical Filters, Macmillan, New York, 1986. 